\title{
Recruitment of patients with lung cancer into a randomised clinical trial: experience at two centres
}

S G Spiro, N H Gower, M T Evans, F M Facchini, R M Rudd, on behalf of the Big Lung Trial Steering Committee breast cancer trial in Scotland ${ }^{2}$ and up to $45 \%$ of patients with lymphoma in British National Lymphoma Investigation studies. ${ }^{3}$

There are many reasons why patients are not entered into trials. Physicians may be unable to find sufficient time to explain the diagnosis, possible treatments, the aims of a study, the concept of randomisation, and then to obtain full written informed consent. Patients invited to participate may decline. In general, it is the younger, more educated patients who appear more willing to consider entry into a randomised controlled trial (RCT). ${ }^{4}$ However, lung cancer affects older people and, more often, those in lower socioeconomic groups.

The Big Lung Trial (BLT) is a multicentre study designed to confirm prospectively the findings of a recent meta-analysis ${ }^{5}$ that cisplatin containing chemotherapy may offer a survival advantage at five years when added to surgery, a small non-significant advantage when added to radical radiotherapy, and a $10 \%$ improvement in survival at one year when added to best supportive care. Once a decision is made regarding appropriate primary treatment, randomisation is to receive or not receive three courses of chemotherapy in addition to the planned primary treatment. The clinician may select from four alternative cisplatin based chemotherapy regimens. The BLT trial design, entry criteria, and follow up data requirements are kept simple and to a minimum. It was hoped that this would permit a considerable increase in recruitment compared with the national average. To assess this, we prospectively identified and followed patients diagnosed with non-small cell lung cancer (NSCLC) at two London centres to determine whether they entered the BLT, and to identify the reasons why they did not. This descriptive exercise may identify ways of improving entry into RCTs.

Conclusions-Despite considerable time and effort, the proportion of patients recruited was small $(9.2 \%)$. Many seen were ineligible but, of 253 potentially eligible patients, $186(73.5 \%)$ refused to enter the RCT.

(Thorax 2000;55:463-465)

Keywords: lung cancer; chemotherapy; randomised controlled trials; patient recruitment

N H Gower, London Lung Cancer Group, CRC and UCL Cancer Trials Centre, $\mathrm{RF}$ and UC Medical School, John Astor House, Foley Street, London W1P 8AN, UK

email: n.gower@ctc.ucl.ac.uk

Received 13 September 1999 Accepted for publication 22 December 1999
The entry of patients into multicentre studies remains unsatisfactory, with less than $5 \%$ of patients diagnosed with lung cancer in the UK entering trials. ${ }^{1}$ There is evidence, however, that if the patient sees an oncologist or visits a unit with a special interest in a malignant disease, the recruitment increases- $12 \%$ in a

\section{Methods}

The study took place at University College London Hospitals NHS Trust between May 1996 and May 1997 and at St Bartholomew's and The London NHS Trust between November 1995 and July 1998. Research staff (NHG, FMF, MTE) identified all patients recently diagnosed as having NSCLC by visiting relevant hospital wards weekly and, where possible, from pathology/cytology records and bronchoscopy lists. Outpatients were identified if they attended the respiratory, radiotherapy, or oncology clinics as new patients. New cases of NSCLC seen in other speciality clinicsfor example, care of the elderly-who were not admitted, investigated, or diagnosed 
Table 1 Summary of findings in 688 patients with newly diagnosed NSCLC considered for entry to Big Lung Trial (BLT)

\begin{tabular}{|c|c|c|}
\hline & No. & Subgroups \\
\hline \multicolumn{3}{|l|}{ Main reasons for ineligibility $(n=435)$} \\
\hline \multicolumn{3}{|l|}{ Logistical } \\
\hline Followed at other centres & 90 & \\
\hline Delay in referral or identification & 71 & $\begin{array}{l}52 \text { Referred too late or slow management } \\
19 \text { Lost to follow up }\end{array}$ \\
\hline \multicolumn{3}{|r|}{ 20 } \\
\hline $\begin{array}{l}\text { Clinical decision not to give } \\
\text { chemotherapy or not to discuss the trial }\end{array}$ & 165 & $\begin{array}{l}72 \text { Frailty/poor performance status } \\
21 \text { Comprehension/language barrier } \\
19 \text { Co-morbidity } \\
16 \text { Inadequate renal function } \\
15 \text { Change in prognosis /condition during } \\
\text { primary treatment } \\
11 \text { Change in diagnosis /unclear pathology } \\
6 \text { Complications with primary treatment } \\
3 \text { Suspected poor compliance } \\
2 \text { Depression }\end{array}$ \\
\hline Clinical decision to offer chemotherapy & 84 & \\
\hline Prior or concurrent malignancy & 25 & \\
\hline \multicolumn{3}{|l|}{ Potentially eligible for BLT $(n=253)$} \\
\hline Patient refused & 186 & $\begin{array}{l}77 \text { No reason given } \\
61 \text { Did not want chemotherapy } \\
23 \text { Family dissuaded patient } \\
9 \text { Did not want involvement in research } \\
8 \text { Wanted chemotherapy } \\
8 \text { Other reasons }\end{array}$ \\
\hline Entered other treatment trials & 4 & \\
\hline Entered Big Lung Trial & 63 & \\
\hline
\end{tabular}

pathologically may not have been identified. To be eligible for the BLT, a patient has to be fit enough to receive chemotherapy, not have a concurrent malignancy or a history of prior malignancy in the preceding three years, be able to give full informed consent, be randomised within 10 weeks of diagnosis or of completing primary treatment (surgery or radical radiotherapy groups), and have a confirmed diagnosis of NSCLC. Clinicians also need to feel that, for a particular individual, there is uncertainty regarding the potential benefit of chemotherapy.

For all patients identified a record was made of sex, date of birth, primary treatment, whether or not they entered an RCT and, if not, the main reason for non-entry. Where multiple reasons for non-entry were recorded for an individual, one main reason was used. During the survey reasons given were grouped together into common categories and are reported here.

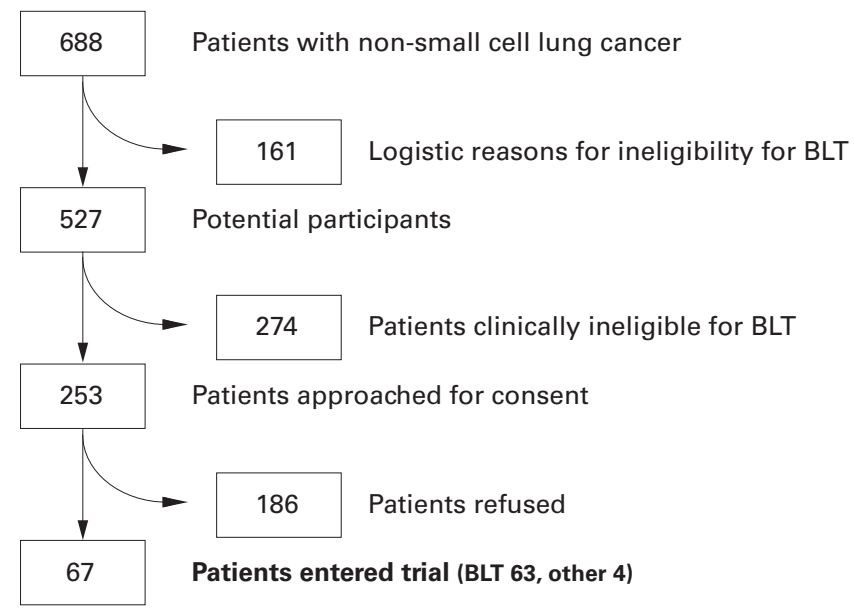

Figure 1 Flow chart of outcome of considering 688 patients with newly diagnosed NSCLC for entry to RCT.

\section{Results}

Six hundred and eighty eight patients were identified with a median age of 67 years (range $32-94) ; 488(71 \%)$ were men. The outcome of considering these patients for the BLT is summarised in table 1 and fig 1 .

Of the 688 patients, $161(23.4 \%)$ were ineligible for logistical reasons. Follow up or further treatment was planned for 90 of these patients at other hospitals not involved in the BLT and so they could not be considered for trial entry. Other logistical reasons for ineligibility occurred when patients were not considered for entry soon enough after diagnosis $(n=52)$ or when patients were lost to follow up ( $n=19)$.

Two hundred and seventy four patients $(39.8 \%)$ were deemed ineligible for the BLT for essentially clinical reasons-for example, clinical decisions not to give chemotherapy included frailty and poor performance status ( $\mathrm{n}=72)$, co-morbid conditions $(\mathrm{n}=19)$, inadequate renal function $(n=16)$, change in prognosis during primary treatment $(n=15)$, or an unresolving complication to their primary treatment $(n=6)$. For 21 patients there were language or comprehension barriers to obtaining consent and a further three patients were felt likely to be poorly compliant with treatment. Two patients were considered to be too depressed for the trial to be discussed. For 11 patients there was a change in their diagnosis during treatment-for example, no malignancy was found at operation or their pathology was unclear.

Although many patients were therefore considered unfit for chemotherapy, 84 patients $(12.2 \%)$ were ineligible because the clinician felt they should be offered chemotherapy. The latter group were younger with a mean age of 58.1 years compared with 66.9 years for the other non-entrants (difference between means 8.8 years, $95 \%$ CI 6.6 to 10.9 ).

Ineligibility due to prior or concurrent other malignancy was recorded in 25 patients $(3.6 \%)$.

Of 253 eligible patients ( $36.8 \%$ of the total), only $63(24.9 \%$ of those eligible) entered the BLT. Four patients entered other treatment trials. One hundred and eighty six eligible patients $(73.5 \%)$ refused to enter the BLT. Refusal rates were highest in the surgical group (83.5\% of those asked) and similar in the radiotherapy $(67.5 \%)$ and best supportive care groups $(68.6 \%)$. Patients were not asked why they were refusing and $77(41.4 \%)$ refused without stating a specific reason. Of those who volunteered a reason, the most common was not wanting chemotherapy (61 patients, $32.8 \%$ ). Only eight patients were recorded as requesting chemotherapy $(4.3 \%)$.

The 63 BLT entrants included a higher proportion of men than the non-entrants $(87.3 \%$ and $69.2 \%$, respectively, $p=0.003, \chi^{2}$ test) and a younger mean age (61.3 and 65.7 years, respectively, difference between means 4.4 years, $95 \%$ CI 1.9 to 6.9 ).

\section{Discussion}

The overall proportion of patients randomised in the BLT was 63 of 688 (9.2\%) although four 
others entered alternative treatment trials. This proportion is higher than the $5 \%$ commonly quoted, but it represents the result of a concerted, organised effort to identify all patients with NSCLC in two large institutions and to spend adequate time discussing the trial with those eligible.

The lessons learnt from this study emphasise many of the problems encountered in entering patients to randomised trials. A large proportion of patients were ineligible for clinical reasons $(39.8 \%)$. General frailty was the commonest reason, which is to be expected in a disease that predominantly affects the elderly and includes patients of all presentations, including advanced disease. The proportion is similar to that quoted with other common cancers. ${ }^{6}$

Despite a reported willingness amongst cancer patients to participate in clinical research, ${ }^{7}$ we could not confirm this view with $73.5 \%$ of eligible patients refusing entry. The highest refusal rate was among patients who had already received potentially curative primary treatment. When told they have lung cancer, patients wish to know their primary treatment and, if it is surgery or radical radiotherapy, their focus tends to be entirely on this. If they are also told of an RCT to consider after the primary treatment, they are likely not to remember when the time comes to decide. ${ }^{8}$ Communication between the respiratory physician, surgeon, radiotherapist, oncologist and patient may not be adequate, and multidisciplinary clinics might overcome this problem. Patients with advanced disease, once informed of their diagnosis and incurability, may not be in a suitable frame of mind to focus on the complexities of an RCT.

We found that the most common reason for eligible patients declining to enter the trial was not wanting chemotherapy. There was considerable concern from patients who had already received a radical treatment which they believed likely to be curative and who did not wish to undergo further potentially toxic treatment of uncertain benefit. Even among those who had received no other specific treatment, many were averse to chemotherapy of which there still appears to be a genuine fear. Statements included: "It frightens me", "I want to die with my hair on", "Chemotherapy is no good for me-it's the treatment that kills people". Family influences were also often explicit with relatives dissuading patients from potentially toxic treatment.

The results of this survey do not concur with previous reports that patients may accept a high degree of toxicity as acceptable in search of cure. ${ }^{9}$ Our results approximate more closely to the findings of Silvestri et $a l^{10}$ who reported that, even after receiving chemotherapy and then being asked whether it was worthwhile for a possible survival advantage of three months, most patients said they would have refused initial treatment. The fact that the two alternatives to which randomisation was sought in the BLT are so different - that is, chemotherapy or no chemotherapy - is probably an important factor affecting the willingness of patients to participate.

Clinical opinions on the value of chemotherapy for NSCLC differ widely, hence the need for the BLT. It is noteworthy that a clinical decision to administer chemotherapy eliminated 84 otherwise eligible patients $(12.2 \%$ of all patients) from participation in the trial although, prior to the publication of the NSCLC meta-analysis, the attitude of 698 physicians treating NSCLC in the UK was extremely negative for the potential of chemotherapy. ${ }^{11}$

Explaining a randomised trial and obtaining informed consent is time consuming and more difficult for the clinician than simply recommending a course of treatment. The clinician may also feel that entry into an RCT may affect the doctor/patient relationship - a view held by $73 \%$ of physicians asked to enter patients into a large trial of surgery for breast cancer. ${ }^{12} \mathrm{~A}$ further $38 \%$ experienced difficulty with the informed consent in that study.

The problems experienced in recruiting to lung cancer trials are similar to those in other malignancies $^{13}$ and perhaps one of the most important elements is time to discuss, explain and listen - an entity of which many doctors have too little. ${ }^{14}$ It has to be hoped that the current national indicators recommending establishment of a multidisciplinary approach for all patients diagnosed with lung cancer will improve their overall care and the potential for entry into RCTs.

1 Stephens R, Gibson D. The impact of clinical trials on the treatment of lung cancer. Clin Oncol 1993;5:211-9.

2 Twelves CJ, Thomson CS, Young J, et al. Entry into clinical Twelves CJ, Thomson CS, Young J, et al. Entry into clinical
trials in breast cancer: the importance of specialist teams. Eur f Cancer 1998;34:1004-7.

3 Hancock BW, Aitken M, Radstone G, et al. Why don't cancer patients get entered into clinical trials? Experience of the Sheffield Lymphoma Group's collaboration in British National Lymphoma Investigation Studies. BMf 1997;314: 36-7.

4 Cassileth BR, Zupkis V, Sutton-Smith K, et al. Information and participation preferences among cancer patients. Ann Intern Med 1980;92:832-6.

5 Non-Small Cell Lung Cancer Collaborative Group. Chemotherapy in non-small cell lung cancer: a metaanalysis using updated data on individual patients from 52 randomised clinical trials. BMF 1995;311:899-909.

6 Ward LC, Fielding JWL, Dunn JA, et al, for the British Stomach Cancer Group. The selection of cases for randomised trials: a registry survey of concurrent trial and non-trial patients. Br F Cancer 1992;66:943-50.

7 Slevin M, Mossman J, Bowling A, et al. Volunteers or victims: patients' views of randomised cancer clinical trials. Br F Cancer 1995;71:1270-4.

8 Penman DT, Holland JC, Bahna GF, et al. Informed consent for investigational chemotherapy: patients' and physicians' perceptions. 7 Clin Oncol 1984;2:849-55.

9 Slevin M, Stubbs L, Plant HJ, et al. Attitudes to chemotherapy: comparing views of patients with cancer with those of doctors, nurses, and general public. BMF
wither with those of doctors

10 Silvestri G, Pritchard R, Welch HG. Preferences for chemotherapy in patients with advanced non-small cell lung cancer: descriptive study based on scripted interviews. BMF 1998;317:771-5.

11 Crook A, Duffy A, Girling DJ, et al. Survey on the treatment of non-small cell lung cancer (NSCLC) in England and Wales. Eur Respir $\mathcal{F}$ 1997;10:1552-8.

12 Taylor KM, Margolese RG, Soskolne CL. Physicians' reasons for not entering eligible patients in a randomised clinical trial of surgery for breast cancer. $N$ Engl $\mathcal{F ~ M e d ~}$ 1984;310:1363-7.

13 Jack WJL, Chetty U, Rodger A. Recruitment to a prospective breast conservation trial: why are so few patients randomised? BMF 1990;301:83-5.

14 Smyth JF, Mossman J, Hall R, et al on behalf of the United Kingdom Co-ordinating Committee on Cancer Research. Conducting clinical research in the new NHS: the model of cancer. BMF 1994;309:457-61. 